\title{
Research on Deepening Path of Agri-product Supply Chain with Blockchain Application
}

\author{
Li Zhang \\ Jinling Institute of Technology, Nanjing 211169, China \\ nj.teacher@163.com
}

\begin{abstract}
China's Agri-product supply chain has various problems such as information asymmetry and lack of trust, while blockchain technology, with its decentralization, security and transparency, and tamper-evident characteristics, will effectively solve the problems of safety traceability and information communication in the Agri-product supply chain. This paper firstly explores the basis of "Dual-chain integration" between blockchain and Agri-product supply chain, then uses game model to study the stability of contractual cooperation between Agri-product supply chain companies, and then explores the deepening path of Agri-product supply chain under blockchain application, to point out the direction for the integration development of blockchain technology and Agri-product supply chain.
\end{abstract}

Keywords: Blockchain; Agri-product Supply Chain; Dual-chain Integration.

\section{Introduction}

The 2020 "No.1 Central Government Document" proposes to accelerate the application of blockchain in the agricultural sector.General Secretary Xi Jinping attaches great importance to the integrated application of blockchain technology and stresses the need to accelerate the development of blockchain technology and industrial innovation.

Through the application of blockchain technology in the field of Agri-product supply chain management, it can help the Agri-product supply chain evolve towards digitalization, intelligence and ecology, and help build a flexible, authentic, real-time and resilient Agri-product supply chain. This paper will analyze the basis of the prospect of "Dual-chain integration" between blockchain and Agri-product supply chain, and then use the game model to study the stability of contractual cooperation in Agri-product supply chain. By exploring the path of deepening the Agri-product supply chain under the application of blockchain, such as creating a new organizational model of "blockchain technology + Agri-product supply chain", the future direction of the integration and development of blockchain technology and Agri-product supply chain is explored to promote the stable operation of Agri-product supply chain.

\section{Foundation for "Dual-chain Integration of Blockchain and Agri-product Supply Chain"}

\subsection{Constraints on Stable Operation of Agri-product Supply Chain}

Agri-product supply chain is a supply chain network system composed of farmers (agricultural products producers), agricultural products traders, agricultural products procurement and processing companies, agricultural products distributors, retailers and logistics distributors and final consumers, and other upstream and downstream companies from field to table.

The high difficulty and cost of logistics has led to a shortage of fresh produce and a continuous rise in prices. The lack of access to information and regulation has prevented effective monitoring of the quality and safety of agricultural products. Min Xu [1] believes that the centralized structure of the Agri-product supply chain is obvious and the node companies are decentralized, which is prone to the problems of information asymmetry and lack of trust, thus leading to low operational efficiency of logistics. Zheng Tan [2] proposed that the main problems of China's logistics supply chain are: the existence of information barriers upstream and downstream, the relationship between cost and 
efficiency is not handled properly, the existence of credit problems as well as the difficulty of financing small and medium-sized companies with large capital needs. There are many participants in the Agri-product supply chain system, involving different fields, different scopes and different subjects, and when they work together to establish production relationships and coordinate production processes, they need to spend high costs to facilitate trust relationships between each other, which include operational costs, document review costs and management costs.

\subsection{Advantages of Blockchain Technology Applied to Agri-product Supply Chain}

Blockchain technology has features such as decentralization, security and transparency, smart contracts and traceability. The data and contracts in the blockchain accept the view and supervision of each node, which not only facilitates the exchange of information but also forms a trustworthy system. Chanjun Li and Haixia Zhang [3] argue that the tamper-evident nature of blockchain improves the security of data, distributed fault tolerance avoids some node errors, and traceability makes it easy to trace and locate various information of commodities. According to Jing Chen [4], in agriculture, the combination of blockchain with the supply chain of agricultural products can effectively reduce the input cost of agricultural products when using the Internet of Things, and can also guarantee the quality and safety of food and foodstuffs. According to Dandan Li [5], the application of blockchain technology to integrate the information of each main body of the Agriproduct supply chain and realize the sharing and integration of information can help optimize the structure and process of the Agri-product supply chain. Existing studies have conducted preliminary analysis on the application of blockchain technology in the Agri-product supply chain, but the role of blockchain technology on the stable operation of the Agri-product supply chain is not yet clear. To this end, the following is an analysis of the contractual cooperation problem in the ecological Agriproduct supply chain based on the game model, and discusses how to ensure the stable operation of the ecological Agri-product supply chain [6].

\section{Game Model Construction for Stable Operation of Agri-product Supply Chain from Perspective of Contractual Cooperation}

\subsection{Game Model Premises for Stable Operation of Agri-product Supply Chain from Perspective of Contractual Cooperation}

This study uses game theory to analyze the decision-making process of knowledge sharing between member companies of Agri-product supply chains and other members. Such decisions are entirely premised on rationality, and supply chain operations are affected when there are problems with the contracts between the subjects of the Agri-product supply chain, thus disrupting the stable operation of the supply chain. To simplify the study, the following premises are proposed:

Premise 1: In an Agri-product supply chain system, only two companies, A and B, are considered to participate, and each seeks to maximize utility.

Premise 2: Both parties to the transaction are finite rational economic subjects.

Premise 3: Company A and B have two choices: default and performance. When one party defaults, given the ease of communication of information within the supply chain, the defaulting party will lose the trust of the rest of the supply chain and will be forced to withdraw from the Agri-product supply chain.

Premise 4: Companies participating in contractual cooperation in Agri-product supply chain are fully autonomous in their decision making, i.e., default and performance.

\subsection{Static Game Model Construction for Stable Operation of Agri-product Supply Chain from Perspective of Contractual Cooperation}

We assume that there are two companies participating in the game of contractual cooperation in the Agri-product supply chain $i(i=1,2)$; the degree of participation of the two companies in 
contractual cooperation is $Q_{i}$; the general benefits that companies obtain without participating in the Agri-product supply chain are $m_{1}$ and $m_{2}$; the coefficients of the ability of Company 1 to obtain benefits from Company 2's performance in the Agri-product supply chain are $R_{C A}$ and $R_{C B}$; only the performing company can obtain the benefits provided by the other enterprises. In addition, we set the coefficient of benefit to be obtained by the contracting company as $P_{i}$, so $P_{i} Q_{i}$ is the benefit obtained by the contracting company. We take the time, resources, potential risk of weakening the competitive advantage of the company and other contingency factors as the cost of contractual cooperation, and assume that the cost coefficient of contractual cooperation is $W_{i}$. $W_{i} Q_{i}$ is the cost of contractual cooperation in the Agri-product supply chain. By comparing the difference in benefits and costs between contractual cooperation and non-contractual cooperation, the cooperating Agri-product supply chain companies make the decision of whether to engage in contractual cooperation. Based on the above research premises, the benefit matrix of the two companies participating in contractual cooperation is shown in Table 1 below.

Table 1. Benefit Matrix of Agri-product Supply Chain Companies Participating in Contractual Cooperation

\begin{tabular}{|c|c|c|}
\hline & Company B (P) & Company B (D) \\
\hline Company A (P) & $m_{1}+R_{C A} Q_{2}+\left(P_{1}-W_{1}\right) Q_{1}, m_{2}+R_{C B} Q_{1}+\left(P_{2}-W_{2}\right) Q_{2}$ & $m_{1}+\left(P_{1}-W_{1}\right) Q_{1}, m_{2}$ \\
\hline Company A (D) & $m_{1}, m_{2}+\left(P_{2}-W_{2}\right) Q_{2}$ & $m_{1}, m_{2}$ \\
\hline
\end{tabular}

\subsection{Analysis of Static Game Model of Agri-product Supply Chain Companies from Perspective of Contractual Cooperation}

According to Table 1, the strategic decision of companies to participate in contractual cooperation in the Agri-product supply chain is directly related to the relevant parameters in the above game model.

Now we will explore the possible strategic decisions of Company A if Company B makes a definite strategic decision of contractual cooperation in the Agri-product supply chain as follows.

If Company $\mathrm{B}$ chooses the contractual cooperation strategy, the benefits obtained by Company A include the benefit obtained by participating in contractual cooperation $m_{1}+R_{C A} Q_{2}+\left(P_{1}-W_{1}\right) Q_{1}$ and the benefit obtained by not participating in contractual cooperation in the Agri-product supply chain $m_{1}$. If $m_{1}+R_{C A} Q_{2}+\left(P_{1}-W_{l}\right) Q_{1}>m_{1}$, i.e., $R_{C A} Q_{2}+\left(P_{1}-W_{l}\right) Q_{1}>0$, Company A may choose to participate in contractual cooperation in the Agri-product supply chain.

If Company B chooses not to pursue the contractual cooperation strategy, the benefits to Company A include the benefit from participating in the contractual $m_{1}+\left(P_{1}-W_{1}\right) Q_{1}$ and the benefit from not participating in the contractual cooperation $m_{1}$. If $m_{1}+\left(P_{1}-W_{1}\right) Q_{1}>m_{1}$, i.e., $\left(P_{1}-W_{1}\right) Q_{1}>0$, Company A may also choose to pursue contractual cooperation in the Agri-product supply chain.

If the strategic decision of contractual cooperation in the Agri-product supply chain of Company A is determined, the strategic decision of Company B is made in the same way as above.

In summary, we conclude the game behaviors of Company A and Company B in the above two cases to create the game matrix of knowledge contractual cooperation behavior and its weighting factors in the Agri-product supply chain shown in Table 2. From the results of the game model discussion, it is easy to understand that if $\left(P_{1}-W_{1}\right) Q_{1}<0$ and $R_{C A} Q_{2}+\left(P_{1}-W_{1}\right) Q_{1}>0$ as well as $\left(P_{2}-W_{2}\right) Q_{2}<0$ and $R_{C B} Q_{1}+\left(P_{2}-W_{2}\right) Q_{2}>0$, companies participating in contractual cooperation of Agri-product supply chain will make the same strategic decisions.

Through the analysis of the above game model, it is easy to see that, under the real situation of trust risk, the contractual cooperation among companies in the Agri-product supply chain is directly related to the gains obtained by companies in the process of contractual cooperation. Despite the existence of information asymmetry and trust deficiency, companies participating in the Agri-product supply chain are willing to engage in contractual cooperation if the gains obtained by cooperative companies can compensate for their losses. 
Table 2. Decision Matrix for Agri-product Supply Chain Companies to Participate in Contractual Cooperation

\begin{tabular}{|c|c|c|c|}
\hline & $\begin{array}{c}\text { Company B } \\
\left(P_{2}-W_{2}\right) Q_{2}>0\end{array}$ & $\begin{array}{c}\text { Company B } \\
\left(P_{2}-W_{2}\right) Q_{2}<0 \\
R_{C B} Q_{1}+\left(P_{2}-W_{2}\right) Q_{2}>0\end{array}$ & $\begin{array}{c}\text { Company B } \\
R_{C B} Q_{1}+\left(P_{2}-W_{2}\right) Q_{2}<0\end{array}$ \\
\hline $\begin{array}{c}\text { Company A } \\
\left(P_{1}-W_{1}\right) Q_{1}>0\end{array}$ & $(\mathrm{P}, \mathrm{P})$ & $(\mathrm{P}, \mathrm{P})$ & $(\mathrm{P}, \mathrm{D})$ \\
\hline $\begin{array}{c}\text { Company A } \\
\left(P_{1}-W_{1}\right) Q_{1}<0\end{array}$ & $(\mathrm{P}, \mathrm{P})$ & $(\mathrm{P}, \mathrm{P})$ & $(\mathrm{P}, \mathrm{P})$ \\
$R_{C A} Q_{2}+\left(P_{1}-W_{1}\right) Q_{1}>0$ & $(\mathrm{D}, \mathrm{D})$ & \\
\hline $\begin{array}{c}\text { Company A } \\
R_{C A} Q_{2}+\left(P_{1}-W_{1}\right) Q_{1}<0\end{array}$ & $(\mathrm{D}, \mathrm{P})$ & $(\mathrm{D}, \mathrm{D})$ & (D, D) \\
\hline
\end{tabular}

\subsection{Role of Blockchain}

The stable operation of Agri-product supply chain depends on the role of factors such as transaction savings within the supply chain, default costs, the benefits of long-term cooperation within the supply chain, and coordination mechanisms between subjects. The impact of blockchain technology on the stability of Agri-product supply chain is also realized by acting on these factors.

According to Chuanheng Sun, Huajing Yu, Daming Xu, Bin Xing and Xinting Yang [7], blockchain technology can effectively solve the trust problem of upstream and downstream data in the transmission process of the supply chain, so as to build a credible transaction environment that matches the traceability demand. According to Haomin Wu, Junjin Chen and Lin Wang [8], the decentralized network distribution and the application of multiple cryptography of blockchain ensure the security of information in the Agri-product supply chain, so the use of blockchain technology can effectively reduce the information asymmetry in the supply chain system and promote the coordination and cooperation between the participants in the Agri-product supply chain.

\section{Deepening Path of Agri-product Supply Chain with Blockchain Application}

\subsection{Create a New Organizational Model of "Blockchain Technology + Agri-product Supply Chain"}

The flattening of Agri-product supply chain organizations should be promoted and various new organizational models for Agri-product supply chains should be created. The integration of blockchain technology and Agri-product supply chain can build a peer-to-peer trading platform and distributed ledger system, and create a flatter form of various Agri-product supply chain organizations by relying on different construction entities. And it can promote the efficient collaboration of agricultural products production, processing, distribution and others.

\subsection{Build a Decentralized IT System of "Blockchain Technology + Agri-product Supply Chain"}

A decentralized information system can be composed of a collection layer, a sharing layer and an application layer. In the collection layer, production information from the production module, transportation and storage information from the logistics module and transaction information from the transaction module are collected in a timely manner, and then enter the sharing layer and communicate with each other. The application layer undertakes various applications of information and provides blockchain-based information services to consumers and relevant departments. The construction of decentralized information system can promote the informationisation and intelligence of the Agri-product supply chain such as production, sales, logistics, finance and supervision. 


\subsection{Create a Consortium for Integration and Development of "Blockchain Technology + Agri- product Supply Chain"}

The process of establishing a sound integration and development of "blockchain technology + Agri-product supply chain" must call for the participation of the government, universities, companies and individual consumers to jointly promote the popularization of blockchain technology. Taking the complementary advantages of resources as the core, the "Dual-chain integration" development consortium is formed to bring together expert teams and companies. It will formulate a roadmap for the development of "Dual-chain integration" technology, form a number of major scientific and technological achievements, formulate a set of technical standards and specifications, and focus on breaking through the underlying application technology in the field of "Dual-chain integration" [9].

\subsection{Develop Talents for Integration of "Blockchain Technology + Agri-product Supply Chain"}

As blockchain technology has not started for a long time, its application in Agri-product supply chain is still immature, and there is a serious shortage of professional talents engaged in the management, technology research and development, network promotion, policy research and data analysis of "Dual-chain integration", so the government should join hands with education departments, research institutions and companies to make more efforts to cultivate Therefore, the government should join hands with education departments, research institutions and companies, and make more efforts to cultivate social organizations and expert teams for "Dual-chain integration" [9], improve the theoretical system for the integration and development of blockchain technology and Agri-product supply chain, and promote the practice of integration and application. It should also encourage talents to go to the front line of the Agri-product supply chain, popularize the theory and experience of blockchain for agricultural products companies and farmers, and help them realize the application of blockchain.

\section{Summary}

The technological features of blockchain provide a new model and method for solving the pain points of the Agri-product supply chain. Therefore, through further improving the organization, basic conditions and deepening path of the integration of "blockchain technology + Agri-product supply chain", we should promote the deep integration of blockchain underlying technology and Agriproduct supply chain, and promote the continuous optimization of Agri-product supply chain. The development and application of blockchain technology is still at an early stage of exploration, but research shows that with the support of blockchain technology, the Agri-product supply chain will be gradually improved, and the strategy of "blockchain + Agri-product supply chain" will be an indispensable step for China to become a strong agricultural country. Blockchain technology is likely to reshape the future development model of Agri-product supply chain.

\section{Acknowledgments}

[Funded Project] This paper is the result of a project entitled "Research on Optimization of Logistics System and Transformation and Upgrading in the Context of the 'Belt and Road' Initiative" (17YJA790027), which was funded by the Humanities and Social Sciences Research Planning Fund of the Ministry of Education.

\section{References}

[1] Min Xu. Analysis of Application Concept and Development Measures of Blockchain in Agricultural Cold Chain Logistics [J]. Logistics Engineering and Management .2019, 41, (05): p. 98-99.

[2] Zheng Tan. Research on Reconfiguration of Logistics Supply Chain from Perspective of Blockchain [J]. Business and Economic Research. 2019, (05): p.83-86. 
[3] Chanjun Li, Haixia Zhang. Application Assumption of Blockchain Technology in Traceability System of Agri-product Quality and Safety [J]. Henan Agriculture. 2019, (17): p. 58-59.

[4] Jing Chen. Research on Logical Architecture of Agri-product Supply Chain based on Blockchain [J]. Modern Agriculture Research.2019 (03): p. 5-7.

[5] Dandan Li. Exploration on Optimization of Agri-product Supply Chain Information with Blockchain Technology [J]. Guangdong Sericulture. 2021, 55 (01): p.122-123.

[6] Jie Shang, Xueqiang Ji. Optimization of Ecological Agri-product Supply Chain with Blockchain Application [J]. Journal of South China Agricultural University (Social Science Edition). 2020, 4: p. 6774.

[7] Chuanheng Sun, Huajing Yu, Daming Xu, Bin Xing, Xinting Yang. Research Progress and Prospect of Blockchain Traceability Technology for Agri-product Supply Chain [J]. Journal of Agricultural Machinery. 2021, 52 (01): p. 1-13.

[8] Haomin Wu, Junjin Chen, Lin Wang. Research on Optimization Path of Agri-product Supply Chain with Blockchain Application [J]. Market Weekly. 2021, 34 (02): p. 41-42.

[9] Chao Zhao. Research on Integration and Development of Blockchain Technology and Agri-product Supply Chain [J]. Xinjiang Finance and Economics. 2020, 5: p. 48-55. 